\title{
HIGH PRECISION CORIOLIS MASS FLOW MEASUREMENT APPLIED TO SMALL VOLUME PROVING
}

\author{
Michael Tombs, Oxford University \\ Manus Henry, Oxford University \\ Feibiao Zhou, Oxford University \\ Robbie M. Lansangan, Invensys Foxboro \\ Michael Reese, Invensys Foxboro
}

\begin{abstract}
This paper discusses small volume provers (SVPs), used in the oil and gas industry to validate the performance of custody transfer meters. Recently Coriolis mass flow meters have been introduced for custody transfer; while these offer reduced maintenance requirements over traditional PD and turbine meters, proving Coriolis flow meters using SVP is challenging. This paper presents SVP results for a Coriolis meter which matches or exceeds the most stringent requirements for custody transfer. This is achieved in part using a novel signal processing technique which reduces the dominant component of the measurement noise, associated with so-called Coriolis mode vibration, with negligible loss of dynamic response.
\end{abstract}

\section{KEY WORDS}

Coriolis Mass Flow metering, Proving, Small Volume Provers, Coriolis mode.

\section{INTRODUCTION}

In the oil \& gas industry, custody transfer is a transaction in which ownership of a quantity of material is transferred from one operator to another, for example, from the well operator to the first transportation operator, via pipeline, truck or ship. Subsequent custody transfer transactions may take place as the crude commodity arrives at the refinery and as refined products leave. The high value of the material, and perhaps the payment of government duties, leads to stringent measurement requirements in order to verify the quantity of material involved in each transaction.

Current standards require flow meters used for custody transfer measurements to undergo regular verification of measurement performance, a process known as meter proving. Commonly this is carried out in situ using a specialized piece of equipment known as a prover, which is based on the principle of filling a known volume. Examples include bidirectional ball provers and compact piston or small volume provers (SVP). Generally, large bi-directional ball provers are used for high volume custody transfer operations. Small volume provers are typically part of a mobile truck-mounted system that travels from site to site proving meters on a scheduled basis.

To carry out proving, the SVP is connected in series with the meter under test. Fig. 1 shows a typical SVP design. Flow passes through a cylinder of known volume. The prover's piston contains a poppet valve which, when open, allows flow to continue irrespective of the piston location; specifically, this allows the piston to be pulled to the upstream position without interrupting flow. With the piston drawn to the upstream position and the poppet valve closed, the piston moves with the fluid as it sweeps through the calibrated volume. At the start and end of each "pass", high precision position detectors generate electrical signals to indicate the passage of the piston. During the pass, the volumetric flow measurement from the meter is integrated, so that a comparison can be made between the known prover volume and the reported volumetric flow from the meter under test.

For a more complete verification of the test meter, proving may be carried out at several flow rates, for example over a 10:1 flow range. At the highest flow rates, a prover pass can be as short as $1.5 \mathrm{~s}$, raising such issues as the noise properties and dynamic response of the flow meter; consequently, the use of electronic or software damping of the measurement output is commonplace. It is also possible to define a "run" at a particular flow rate as consisting of one or more prover passes, where the meter output is summed over all the passes, to increase 
the effective duration of each run. The performance parameters of interest include the repeatability of the meter over a set of runs (each run consisting of one or more passes) at the same flow rate, and the linearity of the meter over the flow range of the test procedure, as explained in more detail below.

Mechanical meters, such as turbine or positive displacement (PD) devices, have traditionally been the most commonly used in liquid custody transfer applications. Consequently the most widely used meter output is the pulse output. This is generated using the blades of a turbine meter, or a gear within a PD meter, which are positioned near a Hall Effect sensor coil. As the blades or gear teeth rotate and pass the pickup coil, a pulse is generated, the output rate of which is proportional to the rotation speed, and hence the flow rate of the fluid, with each pulse representing a discrete volume of fluid. As electronic meters have been introduced, the pulse output has been retained, the microprocessor generating pulses to represent the observed flow rate, thus maintaining compatibility with established standards, practice, and equipment.

One limitation in the use of pulse output is its discrete nature - it is only possible to detect an integer number of electronic pulses; this may place a significant constraint on the repeatability achievable over runs of short duration, given that it is unusual for pulse output frequencies to exceed say $10 \mathrm{kHz}$. An interpolation technique known as double chronometry or the double timing method [2] has been developed to provide an estimate of the fractional number of pulses during a run, thus eliminating the issue of pulse discretisation error.

Over the last ten years, Coriolis mass flow meters have gained acceptance in custody transfer applications, offering reduced maintenance requirements compared with mechanical meters due to the lack of moving parts. In 1996, Whitman described field proving of a Coriolis meter using a small volume prover [3]. Recently, the Weights and Measures Division (WMD) of the National Institute of Standards and Technology (NIST) published a series of articles on the subject of Small Volume Provers [4], including sections on Coriolis meters. The American Petroleum Institute (API) Manual of Petroleum Measurement Standards, Chapter 5, Section 6 on Measurement of Liquid Hydrocarbons by Coriolis Meters [5], provides guidance for the appropriate number of proving runs as well as the number of passes per run for different types of provers, including SVP, in a table, reproduced as table 1. Detailed examination of these documents, however, reveals that guidelines and good practice recommendations are still open to numerous interpretations. For instance, questions often arise as to exactly how many passes per run are permitted, what pulse output damping to use, and what constitutes a minimum acceptable run time.

An early (1998) and detailed examination of the issues associated with on-line calibration of flowmeters using an SVP is provided in the NEL Guidance Note no 7 [6], which describes trials on a range of flow meters, including PD, turbine, ultrasonic and Coriolis meters. Table 2, reproduced from [6], shows the effect of changing the number of passes per run in the NEL trials. This demonstrates the gulf in performance at the time between mechanical and electronic devices for single pass runs, with turbine and PD devices showing repeatability of less than $0.01 \%$, while the ultrasonic and Coriolis meter could only deliver $0.66 \%$ and $0.12 \%$ respectively. Another issue explored in [6] is the delay in pulse output from electronic meters. For example, it proved necessary to delay the gating of the meter output pulses to accommodate electronic and software time constants.

One advantage of a purely mechanical flow system such as a PD meter is that it exhibits negligible delay in the generation of pulses. However, for electronically based devices, measurement noise and microprocessor delay may be detrimental to meter performance, especially on short passes. Of course the use of damping on the pulse output signal reduces noise at the expense of increased delay [7]. Accordingly, proving is considered by some in the industry to be something of a black art, especially when it comes to the setting of the damping factor for a meter that is difficult to prove in the field. Thus Vandiver [8], in offering guidelines on methodologies for Coriolis meter proving, recommends, "The pulse output damping value should never be set higher than 0.8 second." This recommendation is illuminated by the Coriolis meter frequency output signal shown in Fig. 4 of [6], which shows that the pulse output may not have stabilized to a steady flow by the start of the proving run if the damping setting is too high. 
The issues of pulse output delay and damping have become of sufficient concern within the industry that an API Task Group was formed to investigate the Effect of Microprocessor Generated Pulse Output Delay on field proving of meters, including Coriolis and Ultrasonic meters, using SVPs. It is hoped that the current paper will offer some insights into these issues.

The Invensys University Technology Centre for Advanced Instrumentation at the University of Oxford has developed Coriolis metering technology with improved performance characteristics, for example with respect to dynamic response. The reader is referred to the companion paper in this special issue on Coriolis mass flow metering [9] for a background on Oxford's transmitter technology, including the use of Field Programmable Gate Arrays (FPGAs). The importance of the pulse output generation has been addressed through the development of a patented algorithm [10] with high precision (to one part in ten million averaged over one second [11]) and minimal delay.

This technology provides a variety of means for ameliorating what are perceived to be the principle difficulties when calibrating a Coriolis meter using an SVP:

- The first, which is now rare, is the production of irregular pulse streams from the meter. This was found in many early Coriolis meters where changing frequency or missing and adding pulses were techniques used as part of the pulse generation software. This gave rise to uneven or intermittent pulse mark-space ratios. Uneven pulses give rise to poor repeatability when interacting with pulse interpolation, even to the extent that better repeatability can be achieved without using interpolation. In the Oxford transmitter, the $40 \mathrm{MHz}$ clock signal to the FPGA ensures a smooth $50 \%$ duty cycle: even at a high pulse output frequency of $10 \mathrm{kHz}$, there are 2000 clock ticks between edges. This ensures that pulse interpolation techniques will improve repeatability. Of course this technology is entirely immune to the pulse irregularities arising from wear in mechanical meters.

- The second issue arises from damping and time constant. The dynamic response of the meter is pivotal: as previously mentioned, the meter needs to generate a stable output frequency after the initial drop in flowrate caused by the launch of the piston, but before the first detector triggers the start of the pass. Previous trials on the Oxford transmitter [12] have demonstrated that for the bent tube design used in these trials, a very rapid (4ms) step change in flow is indicated via the pulse output from the meter within $16 \mathrm{~ms}$ of the start of the physical change in flow, and that the step change is completed on the pulse output signal within a further $20 \mathrm{~ms}$ or less. For a high-frequency straight flow tube, the companion paper [11] in this special issue reports a delay in the change to the pulse output of less than $4 \mathrm{~ms}$, and insignificant extension of the step change duration. Of course in both these experiments no damping is applied to the pulse output signal as it is considered unnecessary.

- A third issue is also response-time based. Many SVPs show a drop in flow during a pass due to the gas plenum pressure dropping as the piston moves, which must be successfully tracked by the meter. This issue is perhaps best considered as a low frequency flow modulation, as opposed to a step change in flow discussed above. However, as described in the companion paper [11] the Oxford transmitter is able to track $28 \mathrm{~Hz}$ flow modulations. The relatively low frequency flow modulation attributable to pressure drop is easily followed.

Applied to the proving problem, the Oxford transmitter connected to a commercial bent flowtube has passed the most stringent interpretation of current proving standards at a commercial testing laboratory. Specifically, over an 11:1 flow range, covered in eleven steps, using five repeated prover runs each of a single pass only, the meter achieved a repeatability of less than $0.05 \%$ over the range, with an average repeatability of $0.017 \%$. This was achieved without applying any damping on the pulse output signal.

The Oxford research transmitter collected high bandwidth data during the proving runs, and this paper provides a detailed analysis of the data, and describes the signal processing techniques used to provide the measurement performance. Section 2 describes the proving 
trials and results obtained by the testing laboratory. Section 3 provides the corresponding data collected within the Oxford transmitter and demonstrates that they are essentially consistent with those of the testing laboratory. Section 4 provides a more detailed analysis of one set of prover runs and explains how the signal processing techniques employed are able to simultaneously reduce noise with little loss of response time, so that pulse damping is unnecessary. Overall, the aim of the paper is to demonstrate that the latest advances in Coriolis meter signal processing can provide a reliable technology that is able to match the stringent proving requirements of the oil and gas industry.

\section{Proving Trials}

Coastal Flow Measurement, Inc. (CFM), based in Houston, Texas, provides a range of services to the oil and gas industry, including proving facilities via mobile prover skids and a test laboratory in Pasadena, Texas. The laboratory contains a water flow loop with a 75.7 litre (20 US gallon) prover, a Syncotrak Model 25 manufactured by Calibron Systems Inc. The prover volume is calculated using ambient temperature and pressure, with an uncertainty of $0.003 \%$, traceable to NIST. Trials were carried out on a commercial Foxboro $75 \mathrm{~mm}$ (3") Coriolis flow-tube driven by an Oxford transmitter on 31 st January 2005.

The oil and gas industry has a unique system of measurement units which has developed somewhat independently of the scientific measurement community. Accordingly, in this paper measurements will be given in both systems of units as appropriate. One aspect of the oil and gas culture is that, for reasons of historical necessity, commodity measurement has tended to be volumetric, whereas mass, or better yet molar, measurement would provide a more direct assessment of energy content and hence product value. Further elaborate calculations are prescribed to generate "standard" volumes (i.e. corresponding to standard temperature and pressure conditions) which are essentially equivalent to mass measurements. It is ironic that Coriolis meters measure mass flow directly, and yet for the purposes of proving, or indeed oil and gas metering generally, they are required to generate volumetric flow (from the ratio of the mass flow and density measurements) which consequently has a higher uncertainty than the pure mass flow reading; subsequent calculations to generate standard volumes introduce yet further uncertainty in order to return to what is in effect a mass measurement. It is to be hoped that the widening adoption of mass flow measurement will eventually lead to less convoluted oil and gas standards and practices.

The test procedure at CFM consists of installing the meter in the flow loop, and carrying out a series of proving passes at different flow rates. The Oxford transmitter was configured to generate a pulse output of $10 \mathrm{kHz}$ for a volumetric flow rate of 47.32 litres/s (750 US gallons per minute). The $75 \mathrm{~mm}$ flow-tube has a nominal flow rate of $30 \mathrm{~kg} / \mathrm{s}$ or approximately $301 / \mathrm{s}$ of water (approximately 500 US gals $/ \mathrm{min}$ ), and so test flow rates were set at $50,100, \ldots 550$ US gals/min. The laboratory data acquisition system was configured to receive the start and end signals from the prover, and to count the pulses from the meter during each prover pass. Five single pass runs were carried out at each flow rate.

The calculation of repeatability provides one indication of whether the proving results are considered acceptable. The meter factor for the $i^{\text {th }}$ run at flow rate $k$ is defined as

$$
f_{i}^{k}=\frac{\text { prover volume in run } i}{\text { meter volume in run } i}
$$

For a given flow rate, $\mathrm{k}$, the repeatability $\mathrm{R}$, expressed in percent, is calculated as follows:

$$
R^{k}=100 \% \times\left(\operatorname{MAX}_{\forall i} f_{i}^{k}-\underset{\forall i}{\operatorname{MIN}} f_{i}^{k}\right) / \underset{\forall i}{\operatorname{MIN}} f_{i}^{k}
$$

In other words, the repeatability at flow rate $\mathrm{k}$ is proportional to the maximum difference in meter factors at that flow rate over each of the runs (using $\forall i$ to denote over all runs $i$ at flow rate $\mathrm{k}$ ). Note that as required by the API standards, the divisor used is the minimum observed meter factor, rather than its average value. This results in a more stringent test of 
repeatability, but for any reasonable meter performance the difference is marginal. A widelyused proving requirement is that the meter must demonstrate $0.05 \%$ repeatability at each flow rate.

A further performance metric is linearity, defined as follows. The mean meter factor at each flow rate is calculated using

$$
f_{a v e}^{k}=\underset{\forall i}{\operatorname{mean}} f_{i}^{k}
$$

The linearity, $L$, is calculated from the maximum variation in mean meter factor across the range of flow rates under test. Thus:

$$
\left.L=100 \% \times \underset{\forall k}{M A X} f_{a v e}^{k}-\underset{\forall k}{M I N} f_{a v e}^{k}\right),
$$

where $\forall \mathrm{k}$ denotes over all flow rates $\mathrm{k}$. A widely-used proving requirement is that the meter must demonstrate a linearity of less than $0.15 \%$ over the flow range under test. A less stringent requirement of $0.25 \%$ is used in some oil and gas custody transfer applications.

Applying the above definitions, the results for repeatability and linearity were calculated from the meter factors observed by the CFM laboratory data acquisition system over 5 single pass runs at each of the 11 flow steps (approximately $3,6, \ldots 331 / \mathrm{s}$; or $50,100, \ldots, 550$ gals $/ \mathrm{min}$ ). Fig. 2 shows the meter factor for each trial; the average meter factor at each flow rate varies from 1.0005 to 0.9992 , thus yielding an overall linearity of $0.13 \%$, which is within the specified limit for custody transfer $(0.15 \%)$. Fig. 3 shows the repeatability at each flow rate calculated using eqn (2). At the lowest flow rate, the $0.05 \%$ proving limit is marginally exceeded, while at the highest flow rate, the repeatability rises to $0.04 \%$. For all intermediary points, the average repeatability is approximately $0.01 \%$, far exceeding the requirements for custody transfer. The reason for the reduced performance at low flow rate is not clear, although it was the first set of data collected, and shows a relatively large shift in meter factor (Fig 4) compared with the other points. It is possible that the meter had not fully stabilized during this first set of trials. At the highest flow rate, increased flow noise and the short duration of each pass (approximately $2 \mathrm{~s}$ ) offer potential explanations for the reduced performance.

Table 3 shows the complete record collected by CFM for the 200 US gals/min runs; the data values are:

- $\quad$ the duration of each run (column 3);

- the true (traceable) volume dispensed (column 4);

- the total volume recorded from the meter derived from its pulse output during each run (column 5);

- the meter factor (column 6) based on the ratio between columns 4 and 5 , as defined in eqn. 1 ;

- $\quad$ the true volumetric flow rate, based on the ratio of columns 3 and 4 (column 7);

- the total number of meter pulses during the run; this is calculated using double chronometry and is hence fractional (column 10);

- the average pulse output from the meter (column 8), based on columns 10 and 3;

- the meter net gain (column 9) i.e. the average number of pulses per US gallon.

One important feature of the data is that the operation of the prover shows much greater variation in the average flow rate and run duration $(0.3 \%$ repeatability) than in the totalised flow generated from the transmitter $(0.004 \%$ repeatability). This demonstrates the high quality of both the calibration technique (and meter performance) and its suitability for field calibration. Despite the inevitable variations in flow rate due to, for example, pump pressure variations over a relatively short time scale, excellent repeatability is achieved in the total volume dispensed and metered. 


\section{Oxford Transmitter data records}

There are two main types of detailed records that can be collected by the transmitter. One records the raw data from the two flow-tube sensors along with flow-tube temperature information in a so-called "sensor" file, updated at $12 \mathrm{kHz}$. This file type allows the most fundamental reconstruction and analysis of the measurement calculations, but provides no information on other aspects of meter operation, such as flow-tube control or external inputs. The second type of file stores up to 60 channels of operation data in a so-called "record" file. This provides a much wider record of flow-tube behaviour at the measurement update rate i.e. every half-cycle of the flow-tube vibration, typically at $170 \mathrm{~Hz}$. Thus, in a record file the mass flow, density, temperature and volumetric flow measurements are recorded, along with operational parameters such as the frequency and amplitude of oscillation of the flow-tube and the drive current supplied to the flow-tube. Any external inputs are also included in the record file. Both sensor and record files were recorded simultaneously during the proving runs discussed in this paper, in order to provide the widest analysis of meter performance.

In the CFM trials, timed logical inputs were used to record the start and end signals from the prover. The timing of these signals are resolved to better than $1 \mu$ s using dedicated FPGA hardware, and are stored in the resulting record file. It is thus possible to reproduce the results from the proving trials as observed internally by the transmitter, i.e. by integrating the volumetric flow rates between the start and end of each prover pass. This is useful as inevitably the transmission and collection of volumetric flow data via the pulse output leads to increased uncertainty. More importantly, a full data record enables detailed analysis of meter performance which cannot be provided by the test laboratory.

An important first step is to check that the internally recorded data are consistent with the independent record generated by the CFM lab data acquisition system. Fig. 4 shows the volumetric flow data recorded by the Oxford transmitter during the $12 \mathrm{~kg} / \mathrm{s}$ (200 US gals/min) flow run. The start and end signals from the SVP are detected and recorded by the Oxford transmitter, and are denoted on the graph.

The flow profile of each prover cycle is explained as follows. At the beginning of each prover run, the piston is in the downstream position. Pressure from the liquid flow forces the poppet valve partially open, at the cost of increased pressure drop. In this configuration the flow rate is lowest (approximately 3.25 US gal/s, e.g. between $t=0 \mathrm{~s}$ and $\mathrm{t}=3 \mathrm{~s}$ in Fig. 4). When a prover run is initiated, the piston is drawn back to the upstream position once the poppet valve has been fully opened. The resulting flow rate is higher (approximately $3.45 \mathrm{US}$ gal/s, e.g. from $t=3 s$ to $t=12 \mathrm{~s}$ ). As soon as the piston reaches the upstream position the poppet valve is closed mechanically, and the piston moves forwards with the flow. The closure of the poppet valve leads to a small increase in pressure drop and hence a slight reduction in flow rate (approximately $3.35 \mathrm{US} \mathrm{gal} / \mathrm{s}$, e.g. from $\mathrm{t}=12 \mathrm{~s}$ to $\mathrm{t}=20 \mathrm{~s}$ ). As the piston moves, it crosses the detectors to generate the start and end signals for the prover pass. At the end of the run the piston is returned to its default downstream position (e.g. from $t=20 \mathrm{~s}$ to $t=23 \mathrm{~s}$ ) until the next pass begins.

As there is no independent "true" record of the flow profile during the prover runs, it is legitimate to question the validity of the instantaneous flow data shown in Fig. 4. The ability of the transmitter to track step changes in flow has been demonstrated elsewhere (for example [7]), while the nature of the residual "noise" on the flow signal is discussed in section 4 below. However, ultimately it is difficult to envisage how the levels of repeatability demonstrated by the meter could be consistently achieved on such short flow runs unless the instantaneous flow measurement is essentially accurate.

Table 4 provides a comparison of two key parameters for the 12l/s trials as recorded by the Oxford transmitter and the CFM data acquisition system, these being the duration of each batch as denoted by the start and end signals observed by each system, and the total volume recorded by the Coriolis meter, whether observed via its pulse output by the CFM system, or based on internally recorded data. A significant and repeatable discrepancy between the two is the recorded duration of the run duration (columns 3 and 4), with the Oxford transmitter consistently reporting an extra $3 \mathrm{~ms}$ duration for each run. The source of this inconsistency is unclear - both systems should detect the start and end signals to high time precision. 
However, further evidence as to the nature of the inconsistency is provided in Tables 5 and 6 , which shows the corresponding raw data and comparison with the transmitter data for the 24l/s (400 US gals/min) flow run. Here the Oxford transmitter consistently reports an extra $1.5 \mathrm{~ms}$ duration. This suggests that the source of inconsistency is not simply a matter of a fixed time delay in one or other of the systems. One possible explanation is suggested by noting that the time difference in each trial corresponds to the duration of almost exactly four pulses from the transmitter. It is thus feasible that difference in counting and reporting pulses at the beginning and end of the runs between the two systems may explain the discrepancy.

Given this inconsistency, and indeed the generally very small error margins under discussion, it is not surprising that the repeatability results observed from the two systems are similar but not identical. Thus, in Table 4 the CFM system observes $0.004 \%$ repeatability which the Oxford transmitter observes $0.006 \%$ for the 12l/s runs; in Table 6 the corresponding figures are $0.014 \%$ and $0.009 \%$ respectively for $241 / \mathrm{s}$ runs. The CFM system itself has a volume uncertainty of $0.003 \%$, so arguably the $12 \mathrm{l} / \mathrm{s}$ results show marginal consistency between the two sets of observations, whereas the 24l/s results show marginal inconsistency. The conclusion drawn from this comparison is that the internally recorded data shows a broadly similar level of performance to that observed by the laboratory data acquisition system, which nevertheless remains the traceably calibrated arbiter of proving performance. However, it is certainly reasonable to use the internal recorded data as a plausible source of more detailed analysis of the meter performance.

\section{Signal analysis.}

Having demonstrated that broadly speaking the internally recorded data is consistent with that observed via the pulse output, in this section an analysis is provided of the raw sensor data and the signal processing algorithms used to generate these results.

In virtually all Coriolis mass flow meters, the raw sensor data is generated from two sensors (usually velocity-sensing coils), which provide essentially sinusoidal analog signals derived from the motion of the flow-tube. While the primary mode of vibration is at the drive frequency, other modes of vibration are also present in the sensor signal. Indeed, as explained in [7], at least one alternative mode of vibration is necessary for Coriolis forces to manifest a phase difference between the sensor signals indicative of mass flow. A useful simplification of Coriolis meter operation is to consider two modes of vibration - the drive mode, with one mode shape and natural frequency of vibration, and the so-called Coriolis mode, with a different mode shape and natural frequency of vibration. For the purposes of flow measurement, the phase difference observed at the drive frequency is created by flowinduced vibration with the Coriolis mode shape at the drive frequency; the Coriolis mode shape being different to that of the drive mode, it generates equal but opposite contributions to the two sensor signals, and hence a phase difference at the drive frequency.

One of the most basic aspects of Coriolis flow-tube design is the relative placing of the two modes of vibration. The closer they are, the higher the gain of the meter, i.e. the phase difference observed at the drive frequency per unit of mass flow. There may not be much variation possible, especially for straight flow-tube geometries which have highly constrained designs; as discussed in [7], typically the Coriolis frequency is 2.7 times the drive frequency, which leads to relatively low gains, for example a maximum of 0.4 degrees of phase difference over the flow range of the meter. By contrast there is greater flexibility in a so-called bent flowtube design: the natural frequency of the Coriolis mode is within $10 \%$ of the drive frequency in at least one commercial flow-tube design, which consequently exhibits up to 15 degrees of phase difference over its flow range, as demonstrated later in the paper.

However, the benefits of a high gain must be balanced against the signal noise generated by the flow-tube's natural affinity to oscillate at the natural frequency of the Coriolis mode (and indeed any other modes of vibration). A key requirement for any transmitter is to ensure that the meter operates primarily in the designated drive mode and that no other mode of vibration is excited. In extreme or faulty conditions there is at least the theoretical risk that the oscillation control system may excite the wrong mode of vibration. Even in normal 
circumstances for most flow-tubes the sensor signals are inevitably contaminated with low levels of modes of vibration other than the drive frequency. External vibration, step changes in flow, and plant noise generally will all act to stimulate these other modes of vibration which, as will be seen, have a disproportionate effect on the short-term precision of the phase difference calculation. Where the two frequencies are relatively distant from one another (e.g. in a straight tube), conventional low-pass filtering may be sufficient to remove the influence of the Coriolis frequency on the phase difference. As the frequencies are brought closer together, the benefit of the increased gain must be set against the increased difficulty of separating the frequency components corresponding to the two modes of vibration.

For the $75 \mathrm{~mm}$ flow-tube used in these experiments, using water as the process liquid, the drive and Coriolis mode frequencies are approximately $85.6 \mathrm{~Hz}$ and $53.8 \mathrm{~Hz}$ respectively (note that a dual drive design facilitates a drive frequency which is higher than the Coriolis frequency). Fig. 5 shows the power spectral density of one of the sensor signals during the course of the 12l/s proving runs, which consists of a series of sharp peaks rising over a baseline noise floor. The drop in the noise floor of three orders of magnitude, commencing at $180 \mathrm{~Hz}$ and settling at approximately $300 \mathrm{~Hz}$, is achieved using a sixth order low pass elliptical filter. The filtering occurs in the FPGA before the data is passed to the processor, in order to reduce the influence of high frequency components in the measurement calculations.

As demonstrated in the associated table, most of the peak frequencies arise from a multiple of the drive frequency, in some cases with the Coriolis frequency added or substracted. Some clarification is necessary here. Of the peaks identified in the table, only the drive and Coriolis frequencies are natural modes of vibration of the flow-tube (higher modes of vibration also exist, but they are ignored for the purposes of this discussion). All others are, in one sense or another, artefacts. The higher harmonics of the drive mode are generated by non-linearities in the sensor coils and do not represent any physical movement of the flow-tube itself. Other peaks, consisting of some combination of the drive frequency and Coriolis frequency, arise from the action of the flow-tube control system itself. As will be seen shortly, the effect of even very low levels of Coriolis mode component has a large impact on the observed amplitude and phase difference calculations, creating modulations at the beat frequency (i.e. the difference between the drive and Coriolis frequencies, in this case at approximately $31.8 \mathrm{~Hz}$ ). The drive control system in the transmitter responds to the observed modulation in amplitude by adjusting the supplied drive current. This results in corresponding variations in the physical amplitude of the sensor signals at the same frequencies. If the drive control system is switched off, and the flow-tube oscillation is allowed to decay naturally, then the beat frequencies disappear from the sensor amplitude spectrum.

Fig. 6 shows the corresponding phase difference data calculated during the same 12l/s experiments. Two lines are given: the raw and corrected phase differences. Comparison with Fig. 4 shows that the corrected phase difference is the basis for the reported mass flow (and hence volumetric flow) measurements, whereas the raw phase difference trace shows much higher levels of noise, obscuring the step changes in flow associated with the operation of the prover.

The raw phase difference data is calculated using a basic Fourier technique, which calculates phase and amplitude data for each sensor signal at the drive frequency, based on a window of data approximately equal to the period of the drive frequency. The phase difference is obtained simply by subtracting the phase from one sensor signal from that of the other. In the Oxford transmitter, such updates are calculated twice every drive cycle, by overlapping the data windows by $50 \%$, leading to a data update rate of approximately $170 \mathrm{~Hz}$, or roughly every $6 \mathrm{~ms}$.

The Fourier technique has several attractive features, including high precision and immunity to the presence of high harmonics of the selected frequency, but any aharmonic content in the sensor signal can have a significant influence on the resulting phase and amplitude data. This is illustrated by Fig. 7, which shows the power spectra of the raw and corrected phase difference data from Fig. 6 . Two peaks dominate the spectra, at approximately $54 \mathrm{~Hz}$ and $32 \mathrm{~Hz}$, which correspond to the Coriolis frequency and the beat frequency between drive and Coriolis modes, respectively. The primary difference between the raw and corrected signals is 
an order of magnitude reduction in the highest peak at the beat frequency. How has this been achieved?

Conventional (e.g. low pass, band pass or notch) filtering of the phase difference signal will introduce significant phase delay in the measurement signal. This will be detrimental to the measurement performance on SVP runs.

The novel filtering technique exploits the relationship between the effects of the Coriolis mode on the amplitude and phase calculation for each sensor signal. As outlined in Appendix 1, the effect of the Coriolis mode component on the Fourier calculations of the phase and amplitude are proportional to the amplitude of the Coriolis component; however, there is a 90 degree phase shift between the two error terms. Appendix 1 further demonstrates that the calculation of the relative rate of change of amplitude, $\mathrm{r}_{\mathrm{a}}$, results in a parameter that is in phase with, and proportional to, the error induced in the phase calculation arising from the Coriolis component. Here $r_{a}(i)$, the rate of change of amplitude at period I, is defined as:

$$
r_{a}(i)=\frac{a(i+1)-a(i-1)}{a(i)} . f(i),
$$

where $a(i)$ is the Fourier amplitude of the drive frequency calculated at time $i, f(i)$ is the corresponding drive frequency, and time is incremented by the half-period of the drive frequency, as illustrated in Fig 8. Note that the calculation of $r_{a}$ (i) requires knowledge of the amplitude at time (i+1); this in effect introduces a delay of half a period in the measurement calculation, as the transmitter is required to wait for the next amplitude calculation before outputting the corrected phase difference. This typically corresponds to an additional $6 \mathrm{~ms}$ delay, and leads to a total transmitter delay of typically $16 \mathrm{~ms}$. This additional delay has been found in practice to have little detrimental effect on the measurement performance of the meter.

Given that the rate of change of amplitude $r_{\mathrm{a}}$ is proportional to the error in phase calculation caused by the Coriolis mode component, it can be used to provide a correction to reduce this error. The scaling factor between $\mathrm{r}_{\mathrm{a}}$ and the phase error is shown in Appendix 1 to be

$\Psi:=\frac{-1}{2 \cdot f^{2}} \cdot \frac{k}{\sin \left(\pi \cdot \frac{k}{f}\right)}$

The scaled rate of change of amplitude correction factor is applied to the phase estimates calculated from each sensor signal, and then the corrected phase difference is derived from the difference of the two corrected phase values.

The correction assumes that the only source of variation in the calculated amplitude of oscillation is caused by Coriolis mode contamination. Of course in practice there will be genuine change in amplitude, due to variations in flowtube damping and the response of the amplitude control system. However, actual changes in physical amplitude will tend to be observed equally on both sensor signals, while, due to the different Coriolis mode shape, amplitude changes due to Coriolis mode oscillation tend to have equal magnitude but opposite signs on the two sensor signals. Thus, when calculating the phase difference, any rate of change of amplitude correction attributable to actual movement will be cancelled out in the subtraction between the two sensors, while the Coriolis mode correction remains.

The effect of the correction is illustrated in Fig 9, which shows the raw phase difference, amplitude of oscillation, rate of change of amplitude and corrected phase difference for a short period of the same 12l/s set of batches shown in Fig. 4. The time period is selected to cover the first rapid rise in flow rate, at around $3 \mathrm{~s}$, when the poppet valve is fully opened and the piston drawn back to the starting position. Of course this data is not part of batch, but the associated large step in flow is most useful to illustrate the improvement in measurement precision provided by the rate of change of amplitude correction. 
The top graph in Fig 9 shows the raw phase difference data. The measurement update rate is approximately $6 \mathrm{~ms}$, as indicated by the circle symbols. The most striking feature of the raw phase difference data is the Coriolis-mode induced oscillation, occurring at the beat frequency of approximately $31.8 \mathrm{~Hz}$. While it is possible to discern the rise in value occurring between 3.7 and 4.0s, its magnitude is small compared with that of the beat frequency modulation. While a simply low pass filter could suppress the modulation, this would be at the cost of the dynamic response and hence repeatability of the meter.

The second graph in Fig. 9 shows the calculated amplitude of oscillation for sensor 1. It clearly exhibits a similar beat frequency modulation, but closer examination reveals that there is a phase offset between the first and second graphs. For example, at time $t=3.6 \mathrm{~s}$, there is a maximum on the amplitude of oscillation, while the modulation on the phase difference signal is close to a zero crossing (i.e. passing through its mean value). However, the rate of change of amplitude, shown in the third graph, is in phase with the phase difference modulation (note that for sensor 2 the rate of change of amplitude would be 180 degrees out of phase). Applying the correction consists of multiplying the rate of change of amplitude by the scaling constant from equation 6 , and subtracting the result from the sensor 1 phase, applying the corresponding correction to the sensor 2 phase, and then calculating the corrected phase difference from the two sensor phases. The resulting corrected phase difference is shown in the final graph of Fig 9 which demonstrates the significant reduction in beat frequency modulation so that the step change in flow rate is more clearly.

A further benefit of this approach is that it readily adapts to changes in the drive frequency caused by changes in the fluid density. Assuming that a reasonable estimate of the Coriolis frequency is known for any practical drive frequency, then it is possible to adjust the scaling factor $\Psi$ as the drive frequency changes. Other aspects of the solution - for example the 90 degree phase offset between amplitude and phase errors, will be maintained even as the drive and Coriolis frequencies, and hence the beat frequency, change with fluid density.

Finally, to demonstrate that this technique is not restricted to one particular flowtube design, Figs. 10 and 11 shows the raw and corrected phase difference time series and spectra for a different flowtube design, for similar SVP trials at Coastal Flow. Here the flowtube diameter is $100 \mathrm{~mm}$, and the flowrate is approximately $50 \mathrm{l} / \mathrm{s}$ or $800 \mathrm{US}$ gals $/ \mathrm{min}$. The drive and Coriolis frequencies are $80.60 \mathrm{~Hz}$ and $72.57 \mathrm{~Hz}$ respectively. As would be expected from such close frequencies, the phase differences observed are high, rising to 15 degrees. Equally to be expected, the beat frequency is observed in the phase difference data at approximately $8 \mathrm{~Hz}$. The lower the beat frequency, the more significant its influence is likely to be on short batches. However, Figs. 10 and 11 demonstrate that the rate of change of amplitude technique applies equally well to a different set of parameters. By applying a different scaling factor, calculated using equation 6 , the $8 \mathrm{~Hz}$ component is suppressed in the spectrum and the flow profile of the prover runs can be identified in the corrected time series. Despite shorter SVP run durations of only approximately $2 \mathrm{~s}$, the repeatability across this set of 15 runs was recorded by Coastal Flow as $0.03 \%$.

\section{Concluding Remarks}

This paper has describe the performance requirements for custody transfer applications in the oil and gas industry, and demonstrated that the latest generation of Coriolis mass flow meters are able to match those requirements. Insight has been offered into the signal processing issues associated with providing high measurement precision and fast dynamic response, most specifically with the relationship between the drive and Coriolis modes of vibration, and their influence on the raw flow measurement. A noise reduction technique has been described which is helpful in achieving custody transfer levels of performance.

Given the levels of performance that have been demonstrated, consideration can be given to extending current practice in a variety of ways:

- The use of pulse output, with or without interpolation, will eventually become a constraint on precision and/or pass duration. This could be deferred by increasing the pulse output frequency, say towards $100 \mathrm{kHz}$. Certainly this implies no difficulty or loss of precision for FPGA-generated signals [11]. However, this approach brings increasing practical difficulty 
in transmitting and preserving high-frequency signals between the meter and the monitoring system.

- An alternative approach would be to use the computational power of modern transmitters to perform the flow measurement integration internally, providing the prover detection signals as discrete inputs to the meter. This is certainly technically feasible, and would bypass any additional errors introduced by the propagation and collection of the pulse output.

- An interesting question concerns the minimum number of measurement cycles required to obtain a good result. For example, the shortest pass durations described in this paper are approximately $1.5 \mathrm{~s}$, corresponding to approximately 120 drive cycles for this flowtube, or approximately 240 measurement updates. From a practical point of view there seems little benefit from pursuing pass times significantly less than one second. However, in other applications such as batch filling, shorter batch times are desirable, and there is data to suggest acceptable performance over shorter timescales. For example, [13] reports $400 \mathrm{~ms}$ batch repeatability of $1 \%$, where the flow is zero at start and end of the batch; other results are provided for batch times as short as $48 \mathrm{~ms}$ as well as batching to/from empty.

\section{Acknowledgements}

The authors are grateful to Steve Whitman of Coastal Flow Measurement, Inc. and Richard Paton of NEL for detailed technical comments on earlier drafts of the text. The Oxford University research was funded by Invensys and EPSRC. 


\section{APPENDIX 1}

Consider the Fourier calculation of amplitude and phase on a single sensor signal, consisting of the drive signal of amplitude $A$ and frequency $f$, which is contaminated by a Coriolis mode sinusoid of amplitude $B$ and frequency $k$, where $B \ll A$. In this simplified analysis, it is assumed that the true zero crossings of the drive frequency component are identified and used as limits for the Fourier integrals (Fig. 8). As $f$ and $k$ are aharmonic, there is a phase offset $\varphi$ in the Coriolis component which will advance by a constant value from cycle to cycle. For simplicity it is assumed that the phase offset of the drive component is always zero.

In the Fourier method, sine and cosine integrals are calculated, denoted S and $\mathrm{C}$ respectively.

$S:=\int_{0}^{\frac{1}{\mathrm{f}}}[B \cdot \sin [(2 \cdot \pi \cdot k \cdot t)+\phi]+A \cdot \sin (2 \cdot \pi \cdot f \cdot t)] \cdot \sin (2 \cdot \pi \cdot f \cdot t) d t$

It can be shown that

$S:=\frac{A}{2 f}-B \cdot f \cdot \sin \left(\frac{1}{f} \cdot \pi \cdot k\right) \cdot \frac{\cos \left(\frac{1}{f} \cdot \pi \cdot k+\phi\right)}{\pi \cdot\left(f^{2}-k^{2}\right)}$

where the A term is the normal Fourier value and the B term is the (unwanted) influence of the Coriolis mode. The corresponding cosine integral is

$C:=\int_{0}^{\frac{1}{f}}[B \cdot \sin [(2 \cdot \pi \cdot k \cdot t)+\phi]+A \cdot \sin (2 \cdot \pi \cdot f \cdot t)] \cdot \cos (2 \cdot \pi \cdot f \cdot t) d t$

which has an analytic value

$$
C:=\frac{-B \cdot k}{\pi \cdot\left(f^{2}-k^{2}\right)} \cdot \sin \left(\frac{1}{f} \cdot \pi \cdot k\right) \cdot \sin \left(\frac{1}{f} \cdot \pi \cdot k+\phi\right)
$$

Note that the Coriolis components of $\mathrm{S}$ and $\mathrm{C}$ are proportional to $\mathrm{B}$ and modulate with $\sin (\phi)$ and $\cos (\phi)$ respectively.

The phase of the sensor signal at the drive frequency is given by the ratio of the $\mathrm{C}$ and $\mathrm{S}$ integrals. Assuming small angles, so that $\tan ^{-1}(x) \approx x$, the error in phase induced by the Coriolis component is given by

$\psi:=\frac{-2 \cdot B \cdot f \cdot k \cdot \sin \left(\frac{1}{f} \cdot \pi \cdot k\right)}{A \cdot \pi \cdot\left(f^{2}-k^{2}\right)} \cdot \sin \left(\frac{\pi \cdot k+\phi \cdot f}{f}\right)$

while the error in the amplitude (calculated as the root sum square of $C$ and $S$ ) is (to a first order approximation) given by 
$\Delta a:=\frac{-2 B \cdot f^{2} \cdot \sin \left(\frac{1}{f} \cdot \pi \cdot k\right) \cdot \cos \left(\frac{\pi \cdot k+\phi \cdot f}{f}\right)}{\pi \cdot\left(f^{2}-k^{2}\right)}$

The phase and amplitude error terms are closely related but, with sin and cos terms respectively, are 90 degrees out of phase with each other. However, if it is assumed that the only source of amplitude variation is Coriolis mode effects, then the rate of change of amplitude, defined as

$r_{a}(i)=\frac{a(i+1)-a(i-1)}{a(i)} \cdot f(i)$,

where the index $\mathrm{i}$ indicates the cycle over which the Fourier calculation has taken place (see Fig. 8), is in phase with the phase error, having a first order form:

$\Gamma:=4 \cdot f^{3} \cdot B \cdot \sin \left(\frac{1}{f} \cdot \pi \cdot k\right) \cdot \sin \left(\pi \cdot \frac{k}{f}\right) \cdot \frac{\sin \left(\frac{1}{f} \cdot \pi \cdot k+\phi\right)}{A \cdot \pi \cdot\left(f^{2}-k^{2}\right)}$

The ratio of the rate of change of amplitude to the phase error is given by

$\Psi:=\frac{-1}{2 \cdot f^{2}} \cdot \frac{\mathrm{k}}{\sin \left(\pi \cdot \frac{\mathrm{k}}{\mathrm{f}}\right)}$ 


\section{REFERENCES}

[1] International Standards Organisation (ISO) 7278-3 (1999). Liquid hydrocarbons -Dynamic measurement -- Proving systems for volumetric meters -- Part 4: Guide for operators of pipe provers.

[2] International Standards Organisation (ISO) 7278-3 (1998). Liquid hydrocarbons -Dynamic measurement -- Proving systems for volumetric meters -- Part 3: Pulse interpolation techniques.

[3] Whitman, S. K. Operational Experiences Proving Mass Flow Meters with Small Volume Provers, presented at the Energy Week Conference and Exhibition, February 1996

[4] Lee, G. D. Part 1: Small Volume Provers -- Identification, Terminology and Definitions, March 2005; Part 2: Small Volume Provers - History, Design and Operation, June 2005; Part 3: Small Volume Provers (SVPs) - Mathematical Determination of Meter Performance Using SVPs, August 2005, National Institute of Standards and Technology Publications, accessible at the web site www.nist.gov/owm

[5] The American Petroleum Institute (API), "Manual of Petroleum Measurement Standards", Chapter 5, Section 6: Measurement of Liquid Hydrocarbons by Coriolis Meters, available from www.api.org.

[6] NEL, "ON-line Calibration of flowmeters using Small Volume Provers". Flow Measurement Guidance Note Number 7, Feb 1998.

[7] Henry M. et al. The Dynamic Response of Coriolis Mass Flow Meters : Theory and Applications, presented at the 2004 ISA Show Technical Conference Symposium, Houston, TX.

[8] Vandiver, M. Proving Coriolis Flow Meters, presented at the International School of Hydrocarbon Measurement (ISHM), Class CT4130.

[9] Clark et al, "Rapid response of a 'new' coriolis meter", FMI this issue

[10] Henry, M., "Frequency output generation through alternating between selected frequencies", US Patent 6,914,463, Jul. 52005.

[11] Zamora, M., Henry, M., Peter, C., "Generation of frequency output for instrumentation applications using digital hardware", Sensor Review, vol. 23(2), 2003, pp. 143-149.

[12] M. Henry, C. Clark, M. Duta, R. Cheesewright, M. Tombs, Response of a Coriolis flow meter to step changes in flow rate, Flow Measurement and Instrumentation, 14 (2003) pp 109-118.

[13] M. Henry and R. Mercado, "Advances in Coriolis mass flow metering research and technology", atp international, vol 3, pp 24-32. 
Table 1 - Typical Number of Proving Runs (from [4])

\begin{tabular}{|l|l|}
\hline \multicolumn{1}{|c|}{ Proving Method } & \multicolumn{1}{c|}{ Number of Runs } \\
\hline Conventional Pipe Prover & 5 consecutive bi-dir. runs \\
\hline Small Volume Prover & $\begin{array}{l}2-5 \text { runs of multiple passes } \\
\text { each }\end{array}$ \\
\hline Tank Prover & 2 consecutive runs \\
\hline Master Meter & 2 consecutive runs \\
\hline
\end{tabular}




\begin{tabular}{|l|c|c|c|c|}
\hline \multicolumn{1}{|c|}{ Meter Type } & \multicolumn{4}{|c|}{ Number of SVP passes/run } \\
& 1 & 5 & 10 & 20 \\
\hline Turbine & $0.004 \%$ & $0.006 \%$ & $0.008 \%$ & $0.008 \%$ \\
\hline Ultrasonic & $0.660 \%$ & $0.330 \%$ & $0.240 \%$ & $0.180 \%$ \\
\hline Positive Displacement & $0.009 \%$ & $0.007 \%$ & $0.010 \%$ & $0.007 \%$ \\
\hline Coriolis & $0.120 \%$ & $0.040 \%$ & $0.050 \%$ & $0.030 \%$ \\
\hline
\end{tabular}

Table 2 - Effect of number of passes per run on meter repeatability (from [6]) 


\begin{tabular}{|cccccccccc|}
\hline Run & Passes & Run time [s] & \multicolumn{2}{c}{$\begin{array}{l}\text { Volume } \\
\text { dispensed [gal] indicated [gal] }\end{array}$} & Volume & Meter factor & Flow rate & Meter freq [Hz] & Meter net K \\
[gpm] & Meter pulses \\
1 & 1 & 6.007338 & 20.023430 & 20.031310 & 0.999607 & 199.98970 & 1333.789 & 400.15730 & 8012.5230 \\
\hline 2 & 1 & 6.015512 & 20.023430 & 20.030620 & 0.999641 & 199.71800 & 1331.931 & 400.14350 & 8012.2470 \\
\hline 3 & 1 & 5.998312 & 20.023430 & 20.030830 & 0.999631 & 200.29070 & 1335.764 & 400.14770 & 8012.3310 \\
\hline 4 & 1 & 6.003825 & 20.023440 & 20.030640 & 0.999641 & 200.10680 & 1334.525 & 400.14380 & 8012.2560 \\
\hline 5 & 1 & 5.995308 & 20.023440 & 20.031470 & 0.999599 & 200.39110 & 1336.476 & 400.16030 & 8012.5870 \\
\hline \hline & Mean & 6.004059 & 20.023434 & 20.030974 & 0.999624 & 200.09926 & 1334.497 & 400.15052 & 8012.3888 \\
\hline \hline & Rep. & $0.336997 \%$ & $0.000050 \%$ & $0.004244 \%$ & $0.004212 \%$ & $0.337025 \%$ & $0.341234 \%$ & $0.004198 \%$ & $0.004244 \%$ \\
\hline \hline
\end{tabular}

Table 3: data recorded by Coastal Flow during $12 \mathrm{l} / \mathrm{s}$ (200US gal/min) proving runs 


\begin{tabular}{|c|c|ccc|ccc|}
\hline Run & Passes & $\begin{array}{c}\text { CFM batch } \\
\text { duration [s] }\end{array}$ & $\begin{array}{c}\text { Meter batch } \\
\text { duration [s] }\end{array}$ & $\begin{array}{c}\text { Difference } \\
{[\mathrm{s}]}\end{array}$ & $\begin{array}{c}\text { CFM recorded } \\
\text { volume [gal] }\end{array}$ & $\begin{array}{c}\text { Meter recorded } \\
\text { volume [gal] }\end{array}$ & $\begin{array}{c}\text { Difference } \\
\text { [gal] }\end{array}$ \\
\hline 1 & 1 & 6.007338 & 6.010395 & 0.003057 & 20.031310 & 20.031620 & -.000310 \\
\hline 2 & 1 & 6.015512 & 6.018598 & 0.003086 & 20.030620 & 20.031803 & -.001183 \\
\hline 3 & 1 & 5.998312 & 6.001359 & 0.003047 & 20.030830 & 20.031922 & -.001092 \\
\hline 4 & 1 & 6.003825 & 6.006856 & 0.003031 & 20.030640 & 20.032281 & -.001641 \\
\hline 5 & 1 & 5.995308 & 5.998387 & 0.003079 & 20.031470 & 20.030988 & 0.000482 \\
\hline \hline & & Repeatability & & $0.004244 \%$ & $0.006458 \%$ & \\
\hline \hline
\end{tabular}

Table 4: comparison of data recorded by Coastal Flow and by Oxford transmitter for 12l/s (200 US gal/min) runs 


\begin{tabular}{|c|c|c|c|c|c|c|c|c|c|}
\hline Run & Passes & Run time [s] & $\begin{array}{c}\text { Volume } \\
\text { dispensed [gal }\end{array}$ & $\begin{array}{c}\text { Volume } \\
\text { indicated [gal] }\end{array}$ & Meter factor & $\begin{array}{c}\text { Flow rate } \\
\text { [gpm] }\end{array}$ & Meter freq $[\mathrm{Hz}]$ & $\begin{array}{l}\text { Meter net K } \\
\text { [pulses/gal] }\end{array}$ & Meter pulses \\
\hline 1 & 1 & 2.998801 & 20.02359 & 20.03323 & 0.999519 & 400.6320 & 2672.165 & 400.1925 & 8013.291 \\
\hline 2 & 1 & 3.001773 & 20.02359 & 20.03246 & 0.999557 & 400.2354 & 2669.418 & 400.1772 & 8012.986 \\
\hline 3 & 1 & 3.001138 & 20.02360 & 20.03456 & 0.999453 & 400.3202 & 2670.262 & 400.2189 & 8013.826 \\
\hline 4 & 1 & 2.996586 & 20.02360 & 20.03171 & 0.999595 & 400.9284 & 2673.937 & 400.1619 & 8012.683 \\
\hline 5 & 1 & 2.999913 & 20.02361 & 20.03278 & 0.999542 & 400.4837 & 2671.114 & 400.1831 & 8012.110 \\
\hline & Mean & 2.999642 & 20.02360 & 20.03295 & 0.999533 & 400.5199 & 2671.379 & 400.1867 & 8013.179 \\
\hline & Rep. & $0.173097 \%$ & $0.000100 \%$ & $0.014227 \%$ & $0.014208 \%$ & $0.173148 \%$ & $0.169288 \%$ & $0.014244 \%$ & $0.014265 \%$ \\
\hline
\end{tabular}

Table 5: data recorded by Coastal Flow during $24 \mathrm{l} / \mathrm{s}$ (400US gal/min) proving runs 


\begin{tabular}{|c|c|ccc|ccc|}
\hline Run & Passes & $\begin{array}{c}\text { CFM batch } \\
\text { duration [s] }\end{array}$ & $\begin{array}{c}\text { Meter batch } \\
\text { duration [s] }\end{array}$ & $\begin{array}{c}\text { Difference } \\
{[\mathrm{s}]}\end{array}$ & $\begin{array}{c}\text { CFM recorded } \\
\text { volume [gal] }\end{array}$ & $\begin{array}{c}\text { Meter recorded } \\
\text { volume [gal] }\end{array}$ & $\begin{array}{c}\text { Difference } \\
\text { [gal] }\end{array}$ \\
\hline 1 & 1 & 2.998801 & 3.000349 & 0.001548 & 20.033230 & 20.034834 & -0.001604 \\
\hline 2 & 1 & 3.001773 & 3.003303 & 0.001530 & 20.032460 & 20.034050 & -0.001590 \\
\hline 3 & 1 & 3.001138 & 3.002685 & 0.001547 & 20.034560 & 20.033084 & 0.001476 \\
\hline 4 & 1 & 2.996586 & 2.998134 & 0.001548 & 20.031710 & 20.033694 & -0.001984 \\
\hline 5 & 1 & 2.999913 & 3.001442 & 0.001529 & 20.032780 & 20.034192 & -0.001412 \\
\hline \hline & & Repeatability & & $0.014227 \%$ & $0.008737 \%$ \\
\hline \hline
\end{tabular}

Table 6: comparison of data recorded by Coastal Flow and by Oxford transmitter for 25l/s (400 US gal/min) runs 


\section{Captions for Figures}

Figure 1: Small Volume Prover (from [1]). The piston contains a poppet valve allowing flow to continue while the piston is drawn from downstream to upstream. With the poppet valve closed, the piston moves downstream at the same velocity as the liquid.

Figure 2: Meter factor results from prover trials on $75 \mathrm{~mm}$ meter driven by Oxford transmitter, based on five single-pass runs for each flow rate.

Figure 3: Repeatability results from prover trials on $75 \mathrm{~mm}$ meter driven by Oxford transmitter, based on five single-pass runs for each flow rate.

Figure 4: Data recorded by Oxford transmitter during five prover passes, with flow rate of $121 / \mathrm{s}$ (200 US gals/min).

Figure 5: Power spectrum of sensor data for the prover test shown in Figure 4.

Figure 6: Raw and corrected phase difference data for the prover test shown in Figure 4.

Figure 7: Power spectra of raw and corrected phase difference data for the prover test shown in Figure 4.

Figure 8: Overlapping intervals for Fourier-based calculations of amplitude and phase. The zero crossings denote the start and end of each measurement cycle, with a $50 \%$ overlap between consecutive cycles.

Figure 9: Rate of change of amplitude correction during a step change in flow for the prover test shown in Figure 4.

Figure 10: raw and corrected phase difference data during Coastal Flow trials at 50l/s (800 US gals/min) using alternative flowtube design.

Figure 11: power spectra of raw and corrected phase difference data during Coastal Flow trials at 50I/s (800 US gals/min) using alternative flowtube design. 
Figure 1: small volume prover

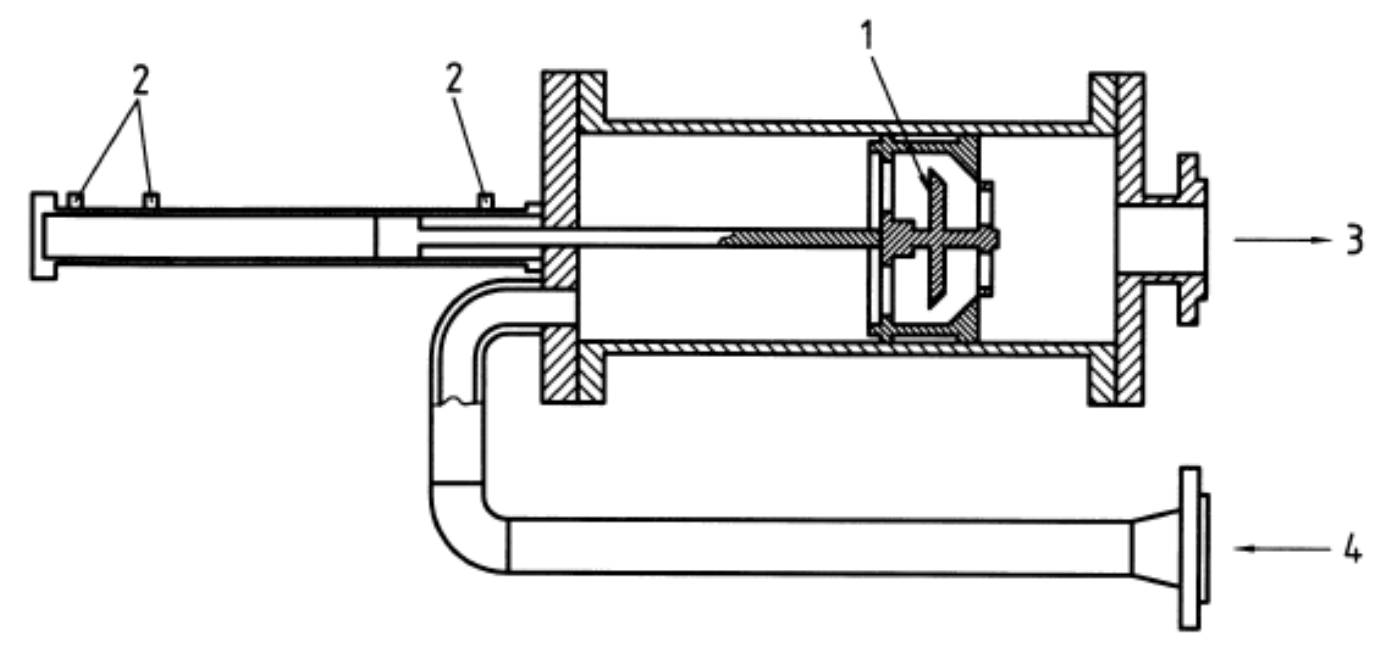

Key

1 Piston and poppet valve arrangement

2 Optical switches

3 Outlet

4 Inlet 
Fig 2. Meter factor results from prover trials on $75 \mathrm{~mm}$ meter driven by Oxford transmitter

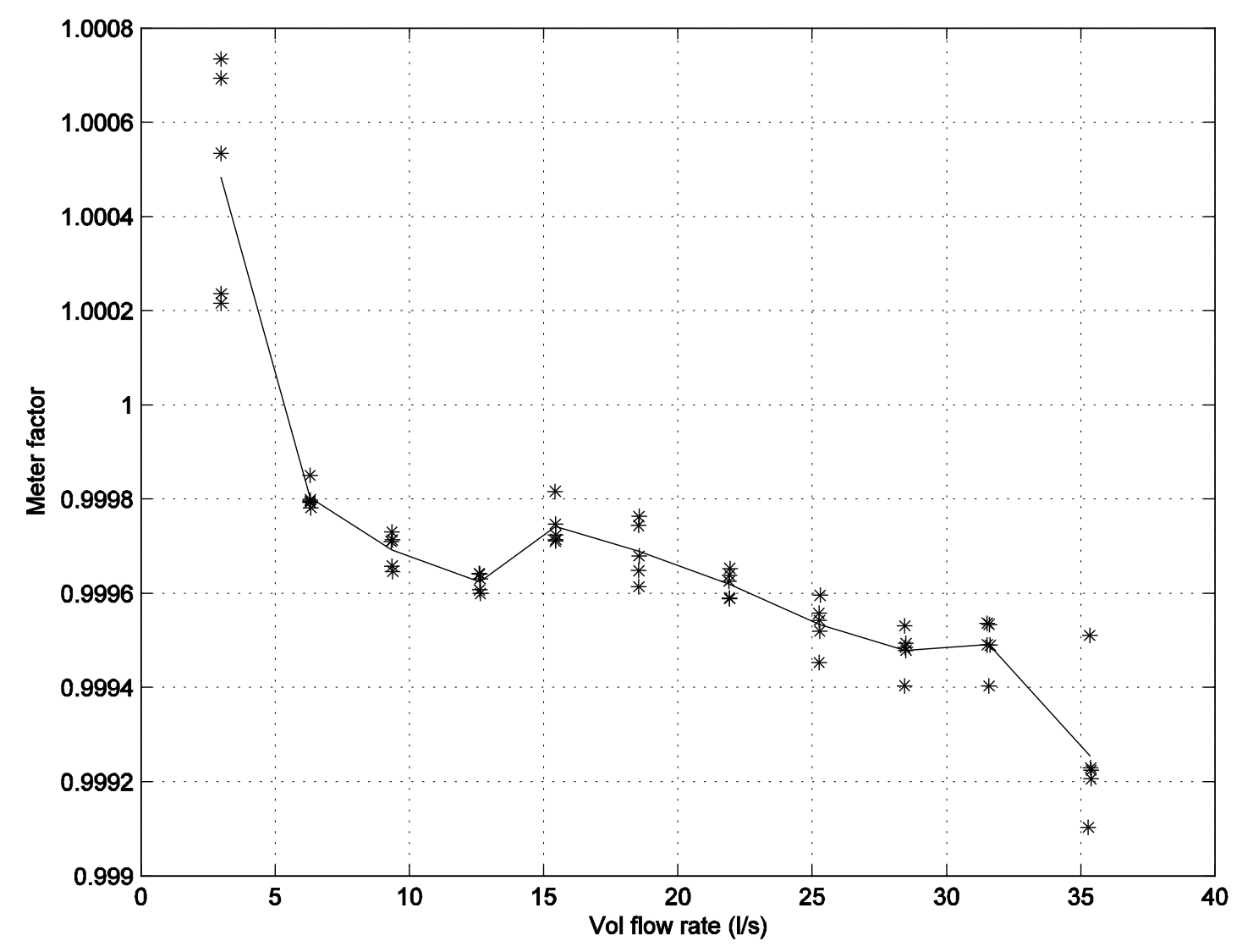


Fig. 3. Repeatability results from prover trials on $75 \mathrm{~mm}$ meter driven by Oxford transmitter

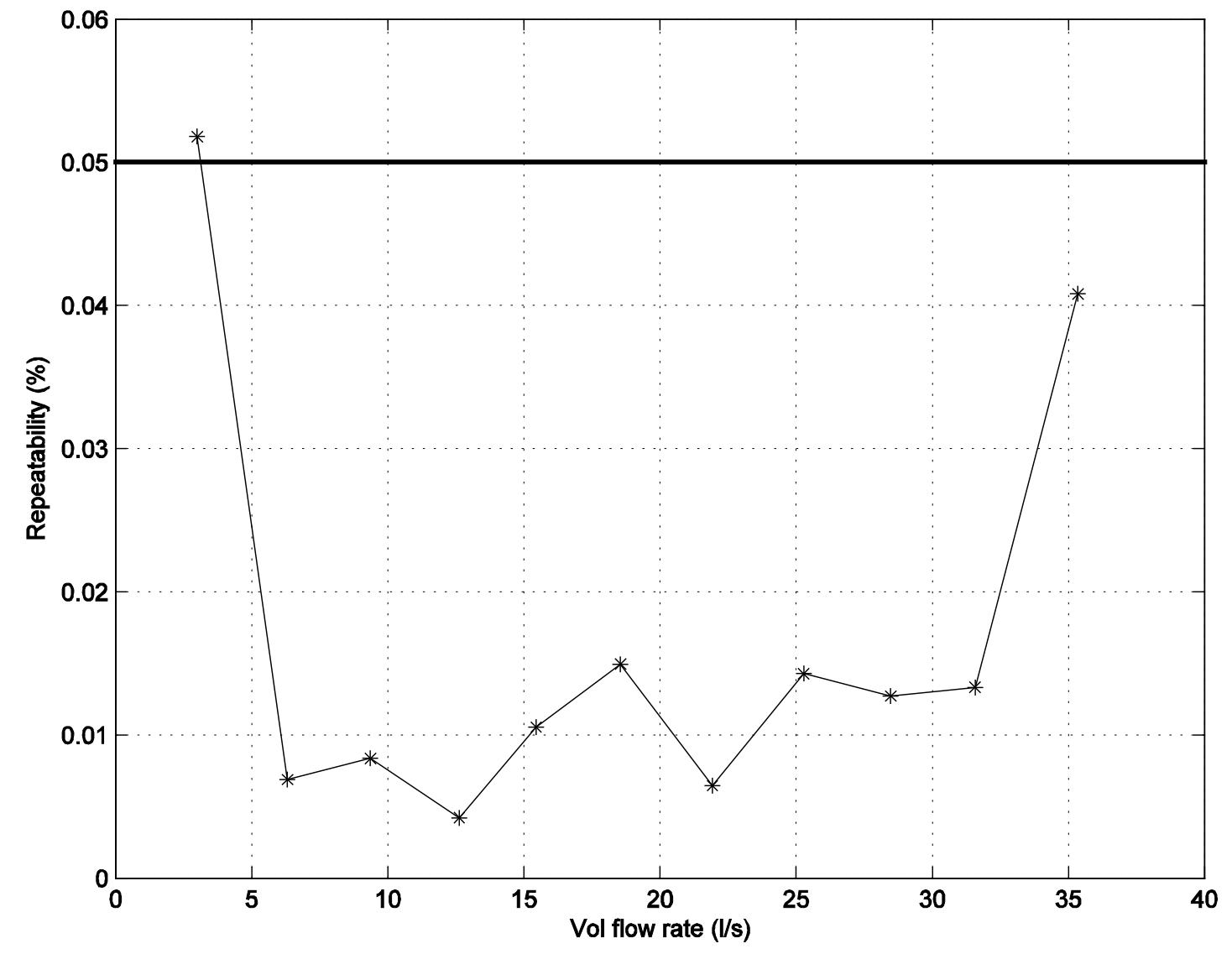


Figure 4: results from Coastal Flow trials at 121/s (200 US gals/min)

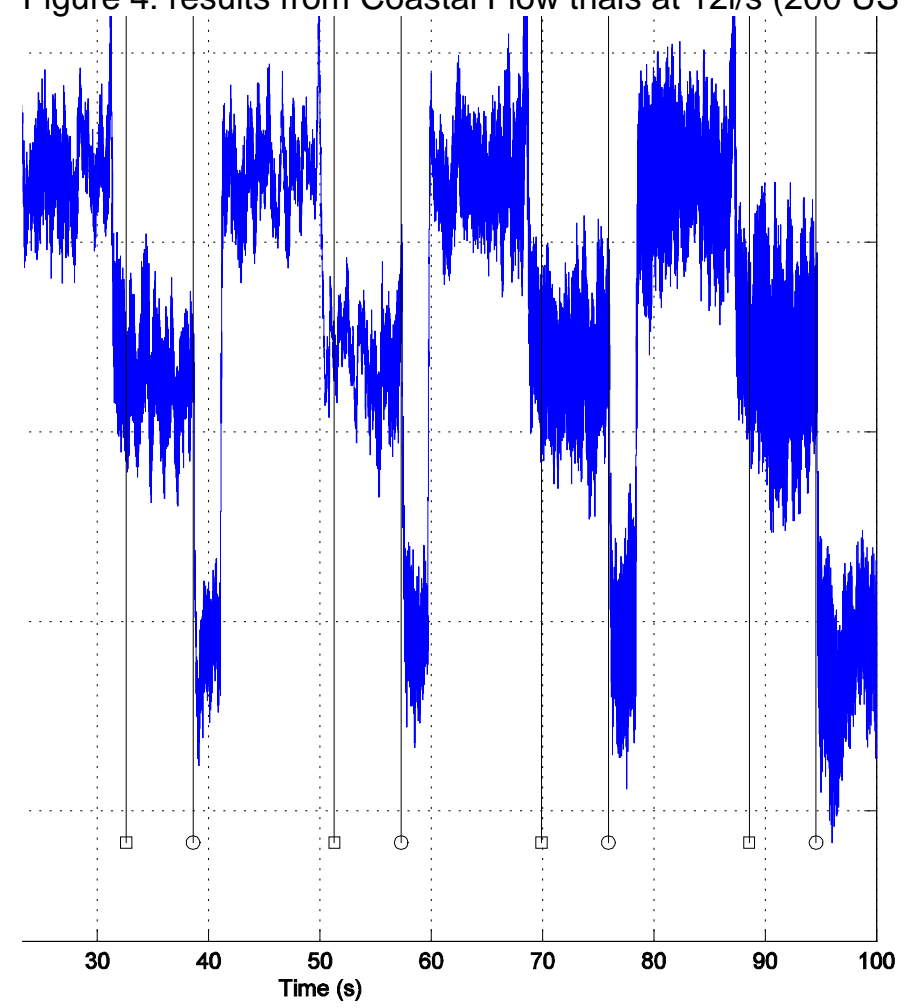


Figure 5: power spectrum of sensor data during Coastal Flow trials at 12I/s (200 US gals/min)

Coastal, FX CFS20 30S, vertical, pure water, 060131-003, filt: 0 FPGA ver: $5.13 \mathrm{P}$ meas rate $12.00 \mathrm{kHz}$ synth rate $48.00 \mathrm{kHz}$

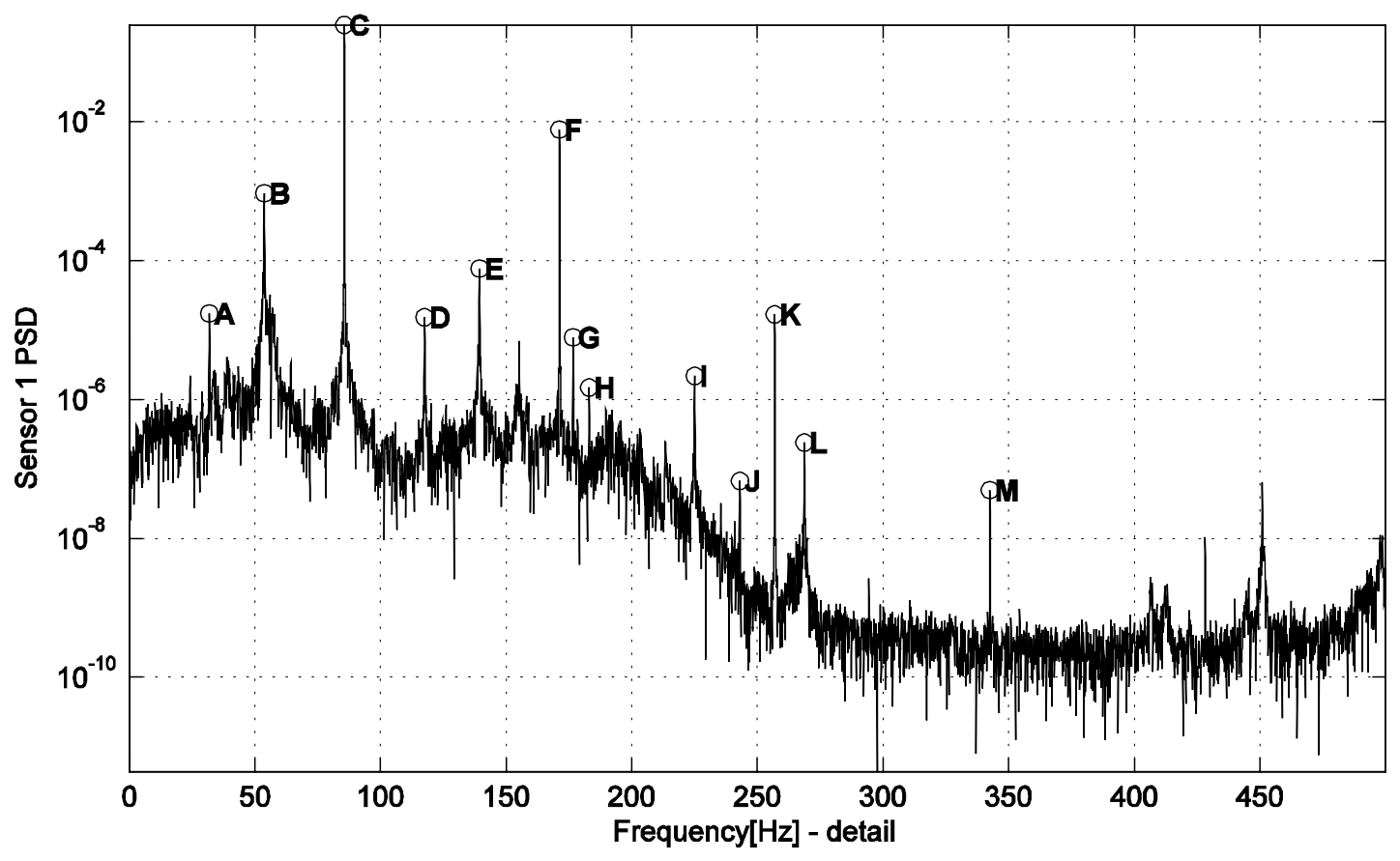

Frequency peak values: (window length: $2.00+00 \mathrm{~Hz}$ )

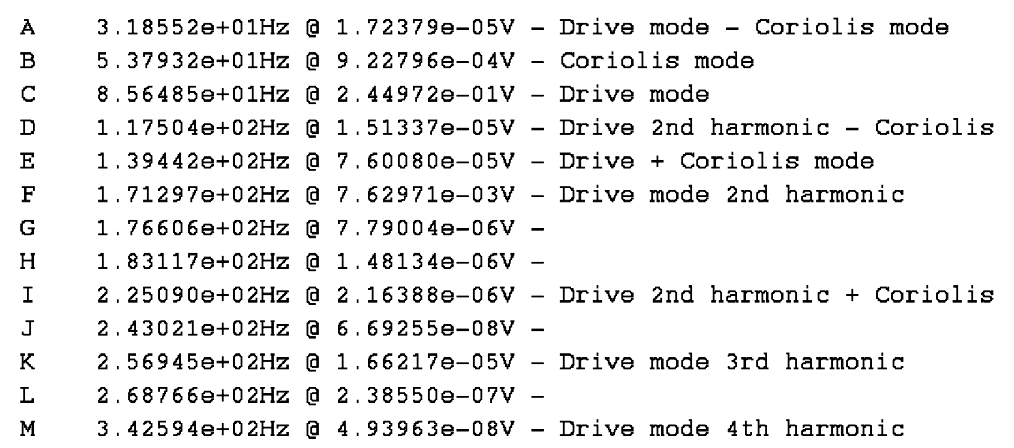


Figure 6: raw and corrected phase difference data during Coastal Flow trials at 121/s (200 US gals/min)

Coastal, FX CFS20 30S, vertical, pure water, 060131-003, filt: 0

FPGA ver: $5.13 \mathrm{P}$ meas rate $12.00 \mathrm{kHz}$ synth rate $48.00 \mathrm{kHz}$

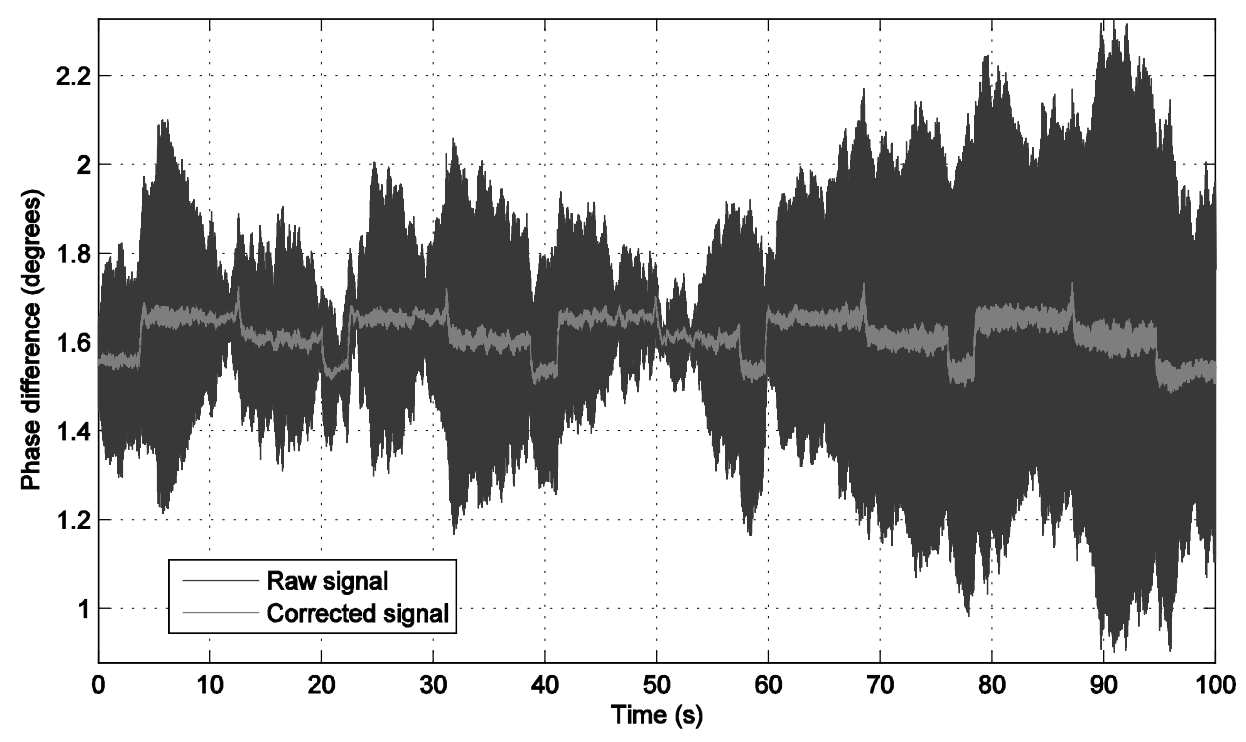


Figure 7: power spectrum of raw and corrected phase difference data during Coastal Flow trials at $121 / \mathrm{s}$ (200 US gals/min)

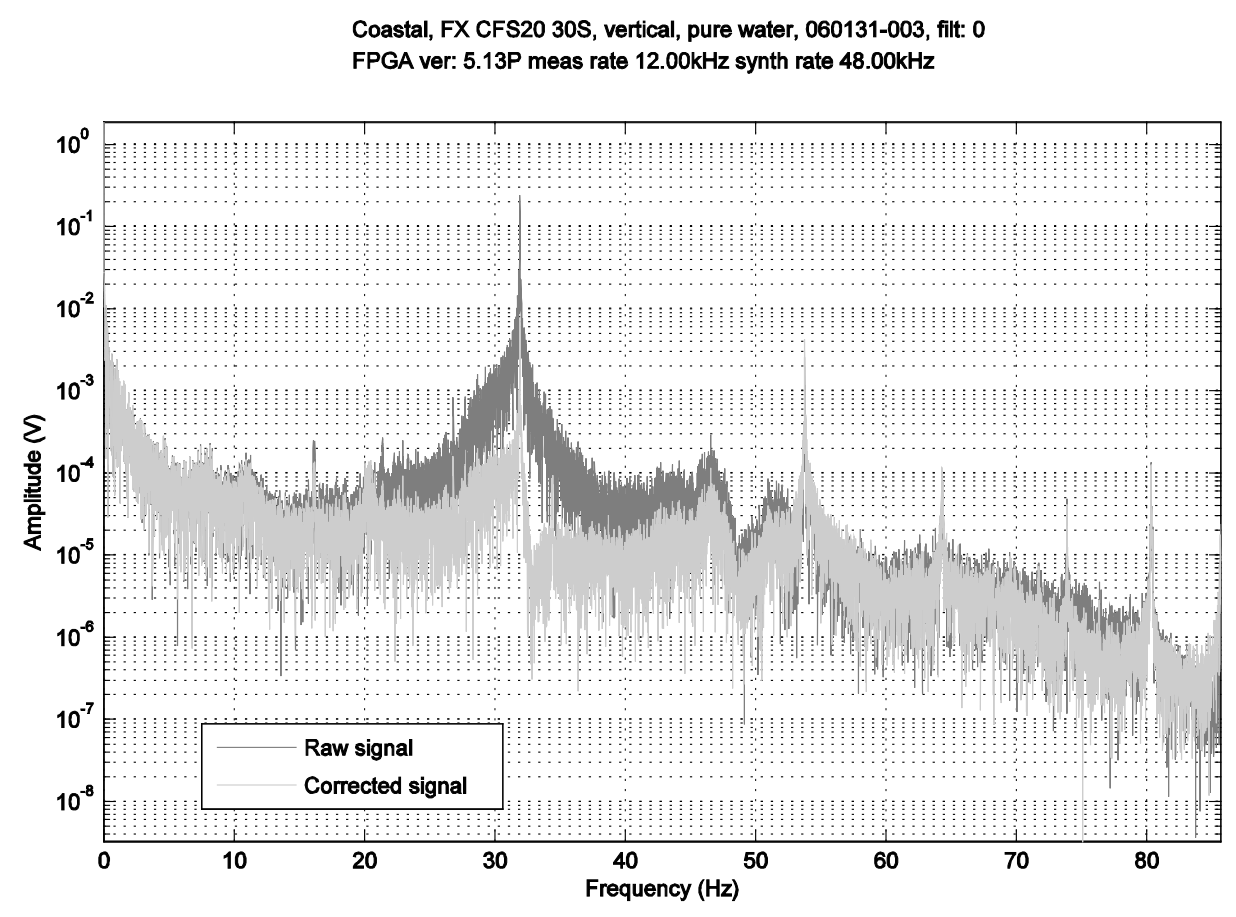


Figure 8: overlapping cycles for Fourier calculations of amplitude and phase

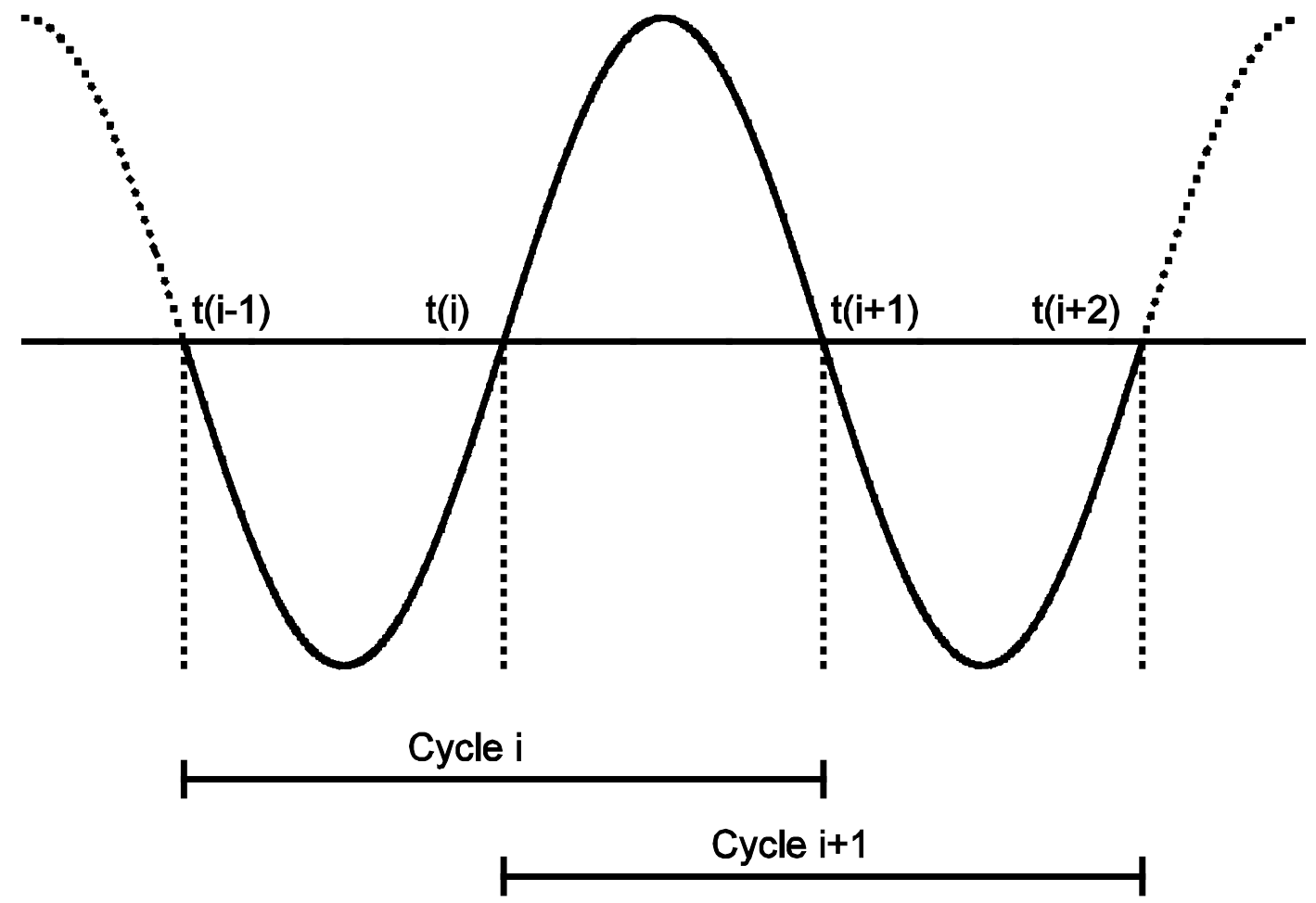


Figure 9: Rate of change of amplitude correction during a step change in flow for the prover test shown in Figure 5.
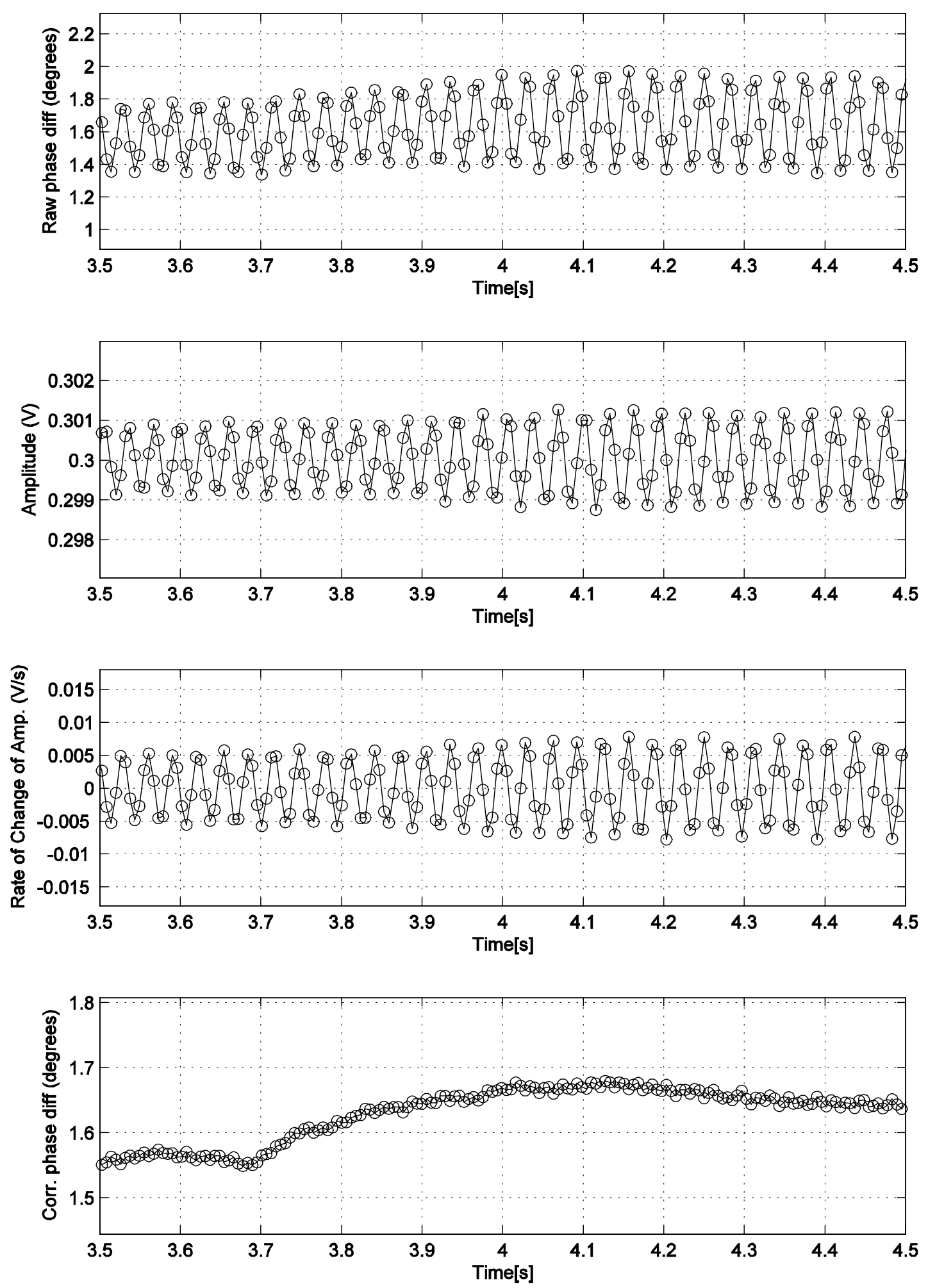
Figure 10: raw and corrected phase difference data during Coastal Flow trials at 501/s (800 US gals/min) using alternative flowtube design

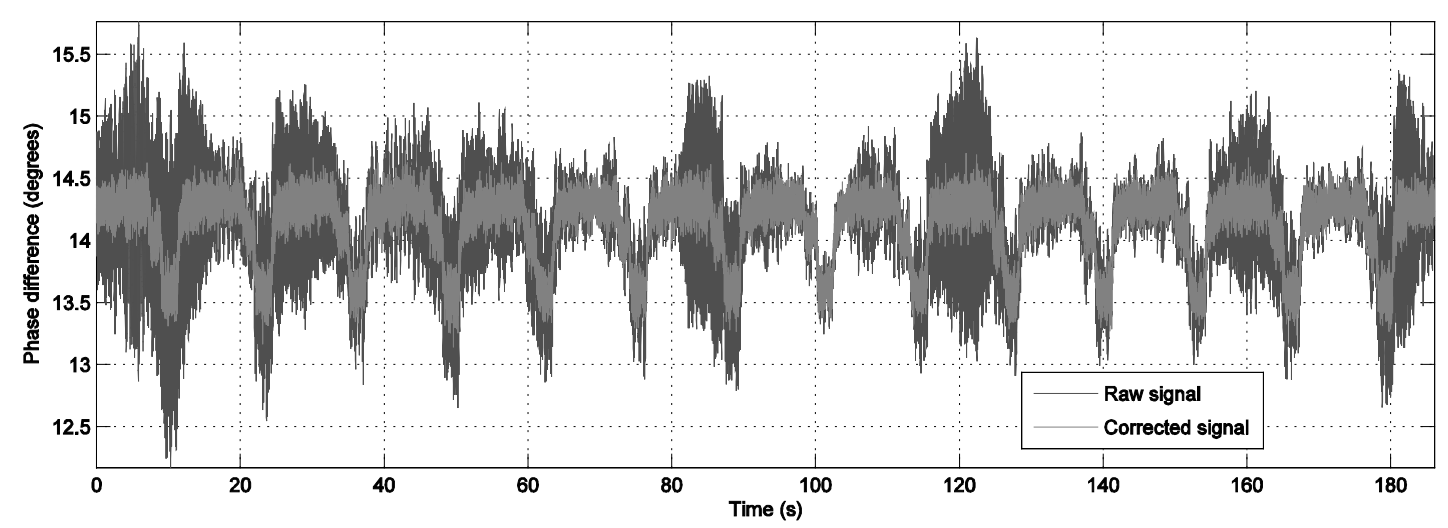


Figure 11: power spectra of raw and corrected phase difference data during Coastal Flow trials at $501 / \mathrm{s}$ (800 US gals/min) using alternative flowtube design

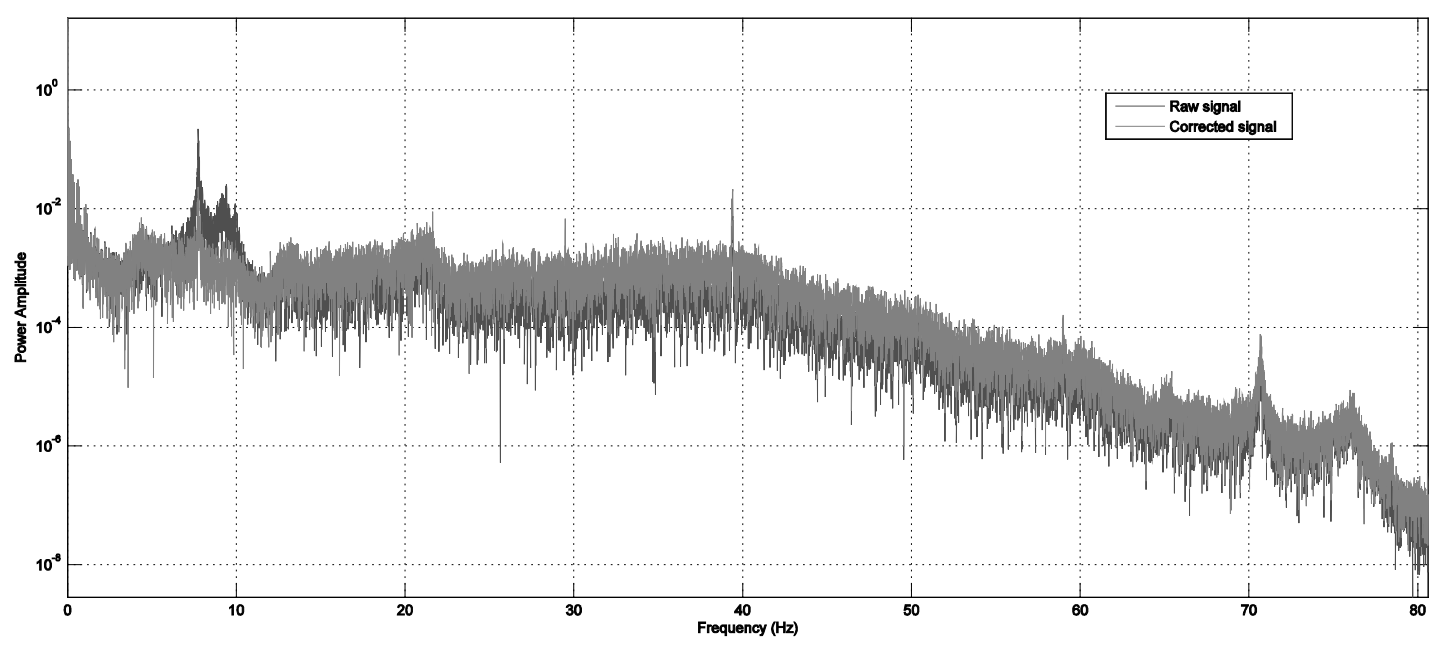

